\title{
Functionalizing silica sol-gel with entrapped plant virus-based
}

\section{immunosorbent nanoparticles}

${ }^{2}$ Global HealthShare ${ }^{\circledR}$ Initiative, University of California, Davis, CA, USA

9 Advancements in understanding and engineering of virus-based nanomaterials (VBNs) for biomedical applications motivate a need to explore the interfaces between VBNs and other biomedically-relevant chemistries and materials. While several strategies have been used to investigate some of these interfaces with promising initial results, including VBN-containing slowrelease implants and VBN-activated bioceramic bone scaffolds, there remains a need to establish VBN-immobilized three dimensional materials that exhibit improved stability and diffusion characteristics for biosensing and other analyte-capture applications. Silica sol-gel chemistries have been researched for biomedical applications over several decades and are well understood;

17 various cellular organisms and biomolecules (e.g., bacteria, algae, enzymes) have been immobilized in silica sol-gels to improve viability, activity, and form factor (i.e., ease of use). Here we present the immobilization of an antibody-binding VBN in silica sol-gel by pore confinement. We have shown that the resulting system is sufficiently diffuse to allow antibodies to migrate in

21 and out of the matrix. We also show that the immobilized VBN is capable of antibody binding and 22 elution functionality under different buffer conditions for multiple use cycles. The promising 
23 results of the VBN and silica sol-gel interface indicate a general applicability for VBN-based

24 bioseparations and biosensing applications.

25

26 Keywords

27 Virus-based nanomaterial; molecular pharming; nanobiotechnology; tobamovirus; plant-made

28 pharmaceuticals; silica sol-gel; bioseparations; biosensing; antibody purification; monoclonal

29 antibody.

30

31 Abbreviations

32 Cy5-TMV, Cyanine 5 conjugated TMV; hIgG, human immunoglobulin G; PEG, polyethylene

33 glycol; PLGA, poly(lactic-co-glycolic acid); TMOS, tetramethyl orthosilicate; TMV, tobacco

34 mosaic virus; TVCV, turnip vein clearing virus. 


\section{Introduction}

36

37

Virus-based nanomaterials (VBNs) are being studied for various medical applications as versatile nanomachines that can be manufactured at an industrial scale with high fidelity and low costs ${ }^{1,2}$. Reports in literature have primarily focused on the design of novel VBNs. With sufficient novelty and value of VBNs having been demonstrated in several application areas, there is a need to consider more advanced VBN-based systems to leverage the potential of existing VBNs and move this technological platform towards the clinic and market.

To date, several studies have explored VBNs as structural and/or functional elements within larger system arrangements. Recent examples include: hot-melt extrusion of trivalent vaccine candidates mixed into slow-release PLGA implants ${ }^{3}$; surface conjugation of osteogenic VBN nanofibers to the surface of 3D-printed bioceramic bone scaffolding ${ }^{4}$; electrostatic layer-by-layer assembly of free-standing VBN biofilms ${ }^{5}$; surface adsorption of immunosorbent VBNs onto gold sensor chips ${ }^{6}$; magnetic particle conjugation to immunosorbent VBNs for protein purification ${ }^{7}$.

Silica sol-gel chemistries represent an alluring set of matrices for bioencapsulation and more advanced VBN-based systems. Extensive literature supports favorable silica sol-gel chemistry characteristics of high structural uniformity, stability, pore size tunability, optical properties, and biodegradability for various biomedical applications ${ }^{8,9}$. A range of live cells ${ }^{10-12}$ and enzymes ${ }^{13-15}$ have been studied for bioencapsulation in silica sol-gel matrices to stabilize viability and activity as well as to improve ease of use for the intended application. There have not yet been any such studies using VBNs, with the exception of a study of viral encapsulation focused on extended release of viral vectors for gene therapy ${ }^{16}$. 
56 In this study we present entrapment and utility of plant virus-based immunosorbent nanoparticles

57 (VINs) in silica sol-gel matrices by pore confinement, representing a novel system configuration

58 for VBNs in general. VINs display antibody-binding proteins on their external coat protein surface,

59 and they have been used as simple and bioregenerable reagents for biosensing and therapeutic

60 antibody purification ${ }^{6,17,18}$. This entrapment of VINs represents the first use of plant virus-based

61 VBNs in a silica sol-gel matrix and the first application of VBN technology for utility in an intact

62 silica sol-gel matrix. We demonstrate that the silica sol-gel matrix can immobilize these large

63 biomolecules over a long-duration ( 30 days) in an environment that preserves their

64 immunosorbent capture and elution functionalities and is sufficiently diffusive for antibodies to

65 freely enter and leave the matrix. We also show that the silica sol-gel encapsulated VINs can be

66 used to purify antibodies from a complex mixture, in this case, crude Nicotiana benthamiana plant

67 extract (representing the production of therapeutic antibodies in plants, formerly known as

68 molecular pharming ${ }^{19}$ ), to overcome reusability and bioprocessing challenges encountered when

69 using VINs in solution to purify antibodies.

\section{2. Materials \& Methods}

\section{$72 \quad 2.1$ Virion production}

73 Turnip vein clearing virus (TVCV) presenting a coat protein display of the D and E domains of

74 Staphylococcus aureus Protein A was used as the plant virus-based immunosorbent nanoparticles.

75 The VINs were produced according to a previously reported study by agroinfiltration of $N$.

76 benthamiana plants using expression vector pICH25892 and processed with a polyethylene glycol 
77 (PEG)-based purification scheme ${ }^{7}$. Final yield was approximately $300 \mathrm{mg}$ VIN per $\mathrm{kg} N$.

78 benthamiana leaf tissue, as determined by Bradford total soluble protein assay.

79 Wild-type tobacco mosaic virus (wt-TMV) was produced via mechanical inoculation of $\sim 5$-week old $N$. benthamiana plants by lightly sprinkling three leaves per plant with Celite ${ }^{\circledR} 545$ (Millipore

81 Sigma, Burlington, MA, USA) as an abrasive aid and gently rubbing $100 \mu \mathrm{L}$ of $0.01 \mathrm{mg} / \mathrm{mL}$ wt-

82 TMV in $0.01 \mathrm{M}$ potassium phosphate buffer $\mathrm{pH} 7.0$ per each of the three leaves. The plant leaves were washed with water 20 minutes after inoculation. Leaf tissue was collected after infection symptoms presented $\sim 1$ week post-inoculation and frozen at $-80{ }^{\circ} \mathrm{C}$ for storage. extraction ratio with $0.1 \mathrm{M}$ potassium phosphate $\mathrm{pH} 7.0$ with $0.1 \%$ (v/v) beta-mercaptoethanol using a chilled mortar and pestle. The plant extract was filtered through three-layered cheese cloth, mixed with equal parts chloroform and n-butanol up to 1:1 (v/v) ratio, centrifuged at 8,000 x $\mathrm{g}$ and $4{ }^{\circ} \mathrm{C}$ for 10 minutes, and the upper aqueous phase layer was collected. PEG-based precipitation was performed by addition of $4 \%(\mathrm{w} / \mathrm{v})$ PEG 8,000 and $1 \%(\mathrm{w} / \mathrm{v}) \mathrm{NaCl}$, incubation of the mixture for 30-60 minutes at $4{ }^{\circ} \mathrm{C}$, and centrifugation at $8,000 \mathrm{x}$ g and $4{ }^{\circ} \mathrm{C}$ for 15 minutes. The pellet was resuspended in $50 \mathrm{mM}$ Tris- $\mathrm{HCl} \mathrm{pH} 7.0$ with a glass rod and let to sit at $4{ }^{\circ} \mathrm{C}$ for $30-60$ minutes.

93 The resuspended solution was centrifuged again at $8,000 \mathrm{x}$ g and $4{ }^{\circ} \mathrm{C}$ for 10 minutes. The resulting supernatant was then ultracentrifuged at 50,000 RPM and $4{ }^{\circ} \mathrm{C}$ for 90 minutes using a 70.1 Ti rotor

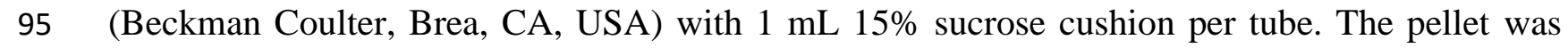
resuspended again in $50 \mathrm{mM}$ Tris- $\mathrm{HCl}$ pH 7.0 with a glass rod and let to sit at $4{ }^{\circ} \mathrm{C}$ overnight. The

97 resuspended solution was added on top of a $10-40 \%(\mathrm{w} / \mathrm{v})$ sucrose gradient and ultracentrifuged at 30,000 RPM and $4{ }^{\circ} \mathrm{C}$ for 90 minutes using a SW40 swinging bucket rotor. A $50 \%$ sucrose solution was used as a plug to fractionate and collect the wt-TMV containing solution. 
Purification of wt-TMV was also performed according to the VIN purification protocol. Final yield was $\sim 800 \mathrm{mg}$ wt-TMV per kg $N$. benthamiana leaf tissue, as determined by UV absorbance A260nm spectroscopy measurements.

The fluorescent reporter particle used in this study, a chemical conjugation of the Cyanine 5 fluorophore to the exterior of the wt-TMV coat protein (Cy5-TMV), was generated using purified wt-TMV and a two-step reaction composed of a diazonium coupling and click reaction step according to a method presented by Bruckman and Steinmetz ${ }^{20}$. Spin filters were used to separate the Cy5-TMV from leftover reaction reagents. Fluorophore dye loading is calculated using extinction coefficients at $260 \mathrm{~nm}$ of $\varepsilon_{\mathrm{Cy} 5}=250,000 \mathrm{~mL} / \mathrm{cm} / \mathrm{mg}^{20}$ and $\varepsilon_{\mathrm{TMV}}=3 \mathrm{~mL} / \mathrm{cm} / \mathrm{mg}^{21}$.

\subsection{Silica sol-gel synthesis}

The silica sol-gel synthesis was performed according to the method for entrapment of liposomes in silica gel detailed by Zeno and team ${ }^{22}$. In brief, $3.8 \mathrm{~mL}$ of tetramethyl orthosilicate (TMOS) was added dropwise to $2.75 \mathrm{~mL}$ of $0.002 \mathrm{M} \mathrm{HCl}$ in a beaker chilled by an ice bath. This mixture was tip sonicated for 15 minutes, added to a round-bottom flask, and rotary evaporated at 340 mbar reduced pressure and $50{ }^{\circ} \mathrm{C}$ for 2-3 minutes before being passed through a $0.22 \mu \mathrm{m}$ filter to yield the final silica sol. The sol was combined with 3 parts PBS by volume and 1 part of a PBS solution containing either VIN, wt-TMV, or Cy5-TMV at $\sim 0.300 \mathrm{mg} / \mathrm{mL}$ concentration (according to total soluble protein measurement for VIN or UV-vis measurement for TMV).

The prepared solution was aliquoted as $40 \mu \mathrm{L}$ droplets (or $2 \mu \mathrm{L}$ droplets for the fluorescent microscope imaging) onto parafilm at room temperature for gel bead formation. Post-gelation $(\sim 1$ hr) beads were submerged in equilibration buffer (PBS pH 7.0) at $4{ }^{\circ} \mathrm{C}$ prior to use. 


\subsection{Binding and elution of human immunoglobulin G}

124 The binding and elution of human immunoglobulin $\mathrm{G}$ (hIgG) with gel beads was performed

125 following a series of processing steps for batch operation: equilibration, sample loading, impurity

126 wash, and elution. Equilibration consists of a $150 \mu \mathrm{L}$ PBS buffer bath to submerge each $40 \mu \mathrm{L}$ gel

127 bead, which was individually contained in a $2 \mathrm{~mL}$ tube, that was nutated at $4{ }^{\circ} \mathrm{C}$ for a minimum

128 duration of 24 hours and exchanged with fresh PBS buffer a minimum of four times throughout

129 the duration. Previous reports in literature have provided 24 hours of equilibration prior to silica

130 sol-gel use ${ }^{23}$, presumably to ensure stabilization of internal pore charges.

131 The hIgG samples were prepared as $80 \mu \mathrm{L}$ of $0.25 \mathrm{mg} / \mathrm{mL}$ hIgG in either PBS or $0.22 \mu \mathrm{m}$ filtered

$132 N$. benthamiana extract. The clarified $N$. benthamiana extract was prepared by $3: 1(\mathrm{v} / \mathrm{w})$ chilled

133 mortar and pestle extraction of 6-week-old $N$. benthamiana leaf tissue frozen at $-80{ }^{\circ} \mathrm{C}$, filtration

134 through 4-layered cheesecloth, centrifugation at $8,000 \mathrm{x} \mathrm{g}$ and $4{ }^{\circ} \mathrm{C}$ for 15 minutes, and an

135 additional filtration through a dead-end $0.22 \mu \mathrm{m}$ syringe filter. For sample loading, all equilibration

136 buffer was removed and the $80 \mu \mathrm{L}$ of sample was added to submerge the silica sol-gel bead, which

137 was kept at $4{ }^{\circ} \mathrm{C}$ nutating for 24 hours.

138 Impurity wash consisted of a minimum of four buffer exchanges into fresh PBS buffer over a 139 period of no less than 24 hours at $4{ }^{\circ} \mathrm{C}$ while nutating.

140 Elution consisted of PBS buffer removal, addition of $80 \mu \mathrm{L} 0.1 \mathrm{M}$ glycine buffer $\mathrm{pH} 2.5$,

141 incubation for 4 hours at $4{ }^{\circ} \mathrm{C}$ while nutating, recovery of the elution solution, and $\mathrm{pH}$

142 neutralization with 0.1 volumes of $0.1 \mathrm{M}$ Tris- $\mathrm{HCl} \mathrm{pH} 9.0$. 


\section{$144 \quad 2.5$ Protein analysis}

145 Protein analyses using methods of the Bradford assay, SDS-PAGE, western blot, and transmission

146 electron microscopy (TEM) were performed according to previously reported methods ${ }^{7}$. UV-vis

147 measurements were made using a Quartz SUPRASIL ${ }^{\circledR}$ quartz cuvette (Hellma Analytics,

148 Plainview, NY, USA) and a SpectraMax ${ }^{\circledR}$ M4 spectrophotometer (Molecular Devices, San Jose,

149 CA, USA). Fluorescence microscopy was performed with an Eclipse 80i microscope (Nikon,

150 Tokyo, Japan) equipped with a 4x objective. Immediately before imaging, Cy5-TMV loaded beads

151 were transferred from a PBS bath to a slide and excess PBS was removed with a pipette. All images

152 were collected using the same exposure time.

153

\section{3. Results}

155 An illustration of the silica sol-gel functionalization with entrapped plant virus-based

156 immunosorbent nanoparticles and the example use case presented in this study is depicted in

157 Figure 1. 


\section{a}

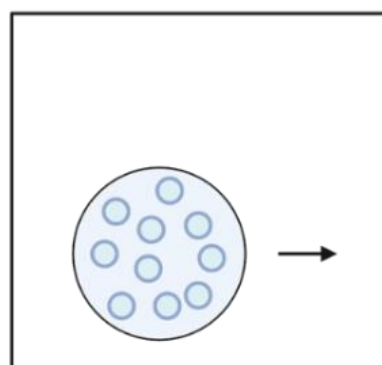

Silica gel precursor

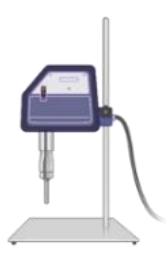

Sonication

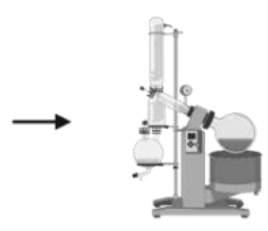

Rotary evaporation

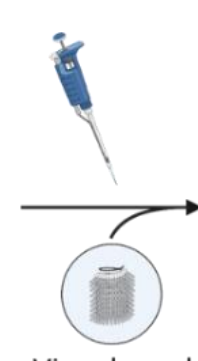

Virus-based nanomaterial
$3 \mathrm{~mm}$
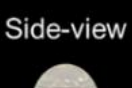

Top-down

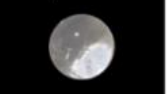

Gelation (40 $\mu \mathrm{L}$ bead shown $)$ b

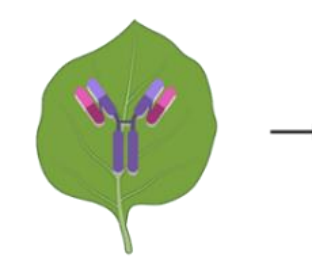

Plant-produced antibody

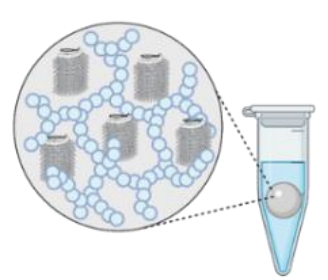

(1) Equilibration

(2) Antibody loading

(3) Impurity wash

(4) Antibody elution

Processing according to plant virus-based immunosorbent nanoparticle procedure

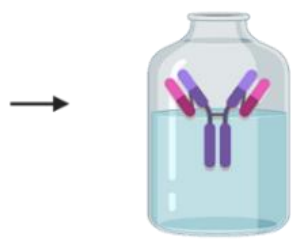

Captured antibody

Figure 1. Illustrated schematics of (a) synthesis of sol-gel functionalized with entrapped virusexample use case presented in this work of gel-entrapped plant virus-based immunosorbent nanoparticles for affinity purification of plant-made antibodies.

Firstly, a fluorescent reporter VBN was developed to investigate silica sol gel entrapment. As of purity. We then generated Cy5-TMV as a reporter system using previously reported methods to conjugate Cyanine 5 fluorophore to the exterior surface of wt-TMV coat ${ }^{20}$. UV-Vis absorbance spectroscopy 200 - $700 \mathrm{~nm}$ spectrums of wt-TMV and Cy5-TMV are comparable to the spectrum the wt-TMV include the introduction of an absorbance peak at around $324 \mathrm{~nm}$, which is consistent 
171 with the introduction of a diazonium bond (needed for the alkyne addition reaction), and another

172 peak at around $646 \mathrm{~nm}$, which is consistent with the sulfo-Cy5 azide absorbance. We estimate a

173 dye loading of $\sim 32 \%$, which is to say that on average $\sim 680$ of an estimated 2,130 coat proteins per

174 assembled virion were conjugated with a Cy5 fluorophore on the exterior surface. Negative stain

175 TEM images were used to confirm structural integrity of the Cy5-TMV (Figure 2c).

176 Plant virion entrapment due to crosslinking physical immobilization during silica sol-gel bead

177 synthesis was then assessed using the Cy5-TMV reporter system. Normalized areal fluorescence

178 measurements are shown over the course of a 48-hour study in Figure 2d. Representative

179 fluorescence microscopy images used in the quantitative measurements are shown in Figure 2e.

180 Fluorescence results indicate that the initial Cy5-TMV concentration in the silica sol-gel matrix

181 was maintained throughout the period of examination. There were appreciable decreases in gel

182 bead volume due to minor breakages and shrinkage over the course of multiple microscope images

183 (Supplementary Information, Figure S1). We attribute this observed behavior to the small bead

184 size $(2 \mu \mathrm{L})$, forceps manipulation, and multiple exposures to a dry environment used for

185 fluorescent microscope imaging. This observation of areal shrinkage was not noted in the beads

186 used for functional testing $(40 \mu \mathrm{L})$.

187 There was no evidence of VIN or wt-TMV lost in the PBS wash buffer during standard 188 equilibration to suggest incomplete entrapment. However, we performed an experiment using

189 lower wash buffer volumes (1 vol buffer: 1 vol gel) to improve the limit of detection and found 190 that $<10 \%$ of the initial VIN or wt-TMV mass added to the gel was recovered in the wash buffer 191 during equilibration, peaking at an initial brief wash timepoint and decaying rapidly from there 192 (Supplementary Information, Figure S2). This suggests that a small amount of the VIN or wt-TMV 

solution.

a

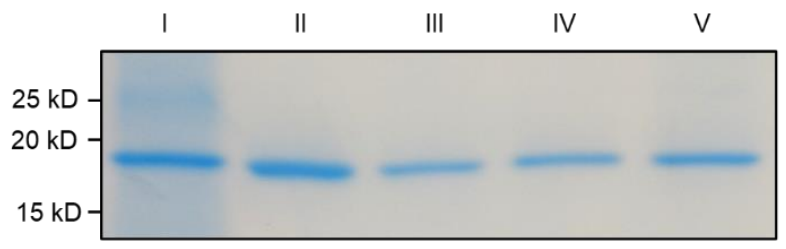

C
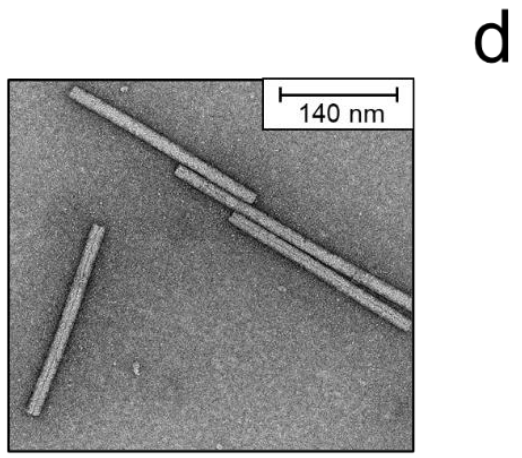

b
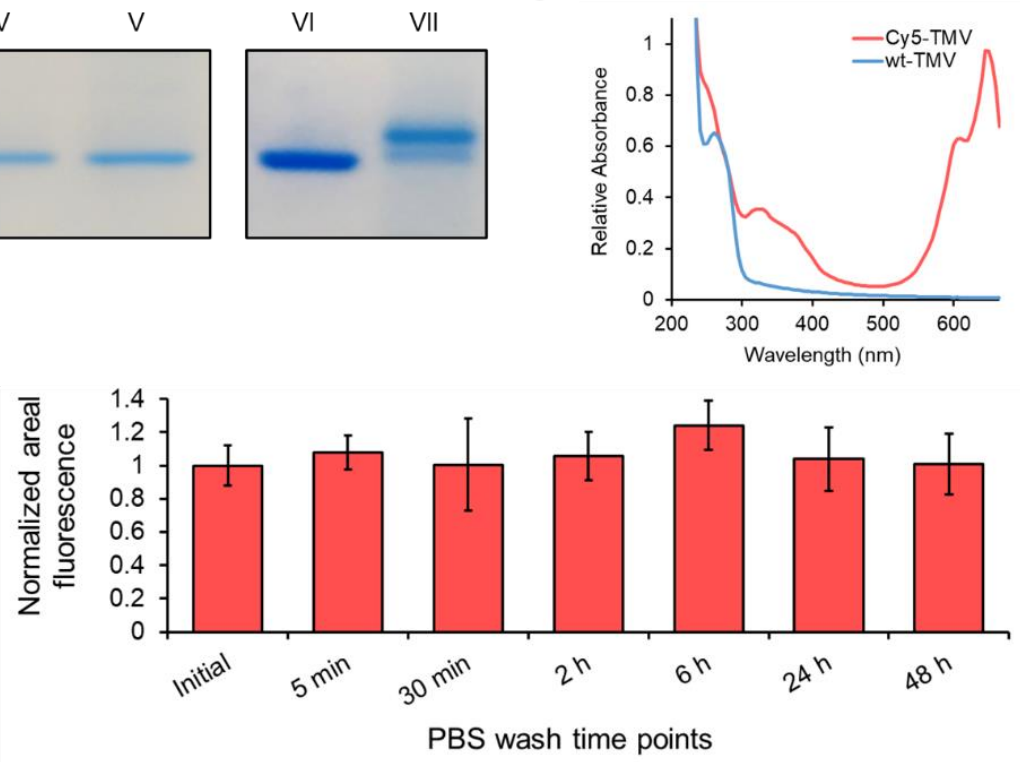

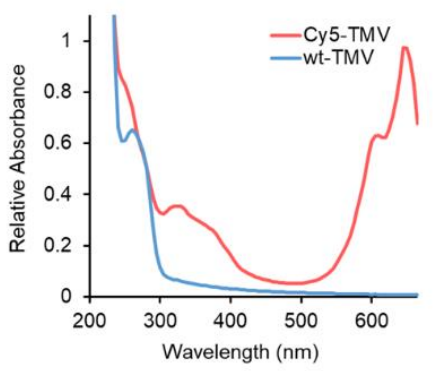

195

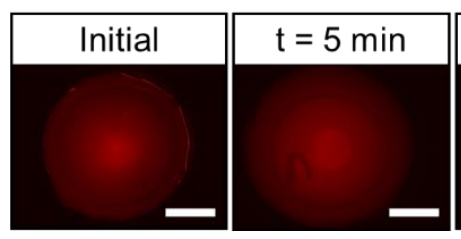

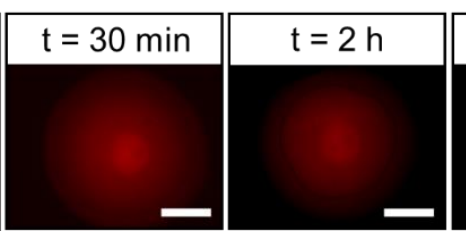

Figure 2. Purification and immobilization of fluorescently labelled plant virions (a) SDS-PAGE results of an ultracentrifuge-based purification of wt-TMV from Nicotiana benthamiana leaf tissue, which is then chemically conjugated with Cy5 dye in a two-step reaction to generate Cy5TMV. Lane definitions: I - filtered plant extract, II - PEG-precipitated pellet resuspension, III microfuge pellet, IV - ultracentrifuge pellet resuspension, V - ultracentrifuge wash, VI - initial wt-TMV pre-conjugation, VII - Cy5-TMV post-conjugation. (b) UV-Vis absorbance spectra of wt-TMV and Cy5-TMV. (c) Negative stain transmission electron microscope image of the Cy548 hours. Beads were exchanged into fresh PBS buffer after each measurement. Error bars 
represent one standard deviation with biological triplicate. (e) Fluorescent microscope images of the silica sol-gel beads containing Cy5-TMV over 48 hours of submerged gel wash. Scale bars represent $500 \mu \mathrm{m}$.

a

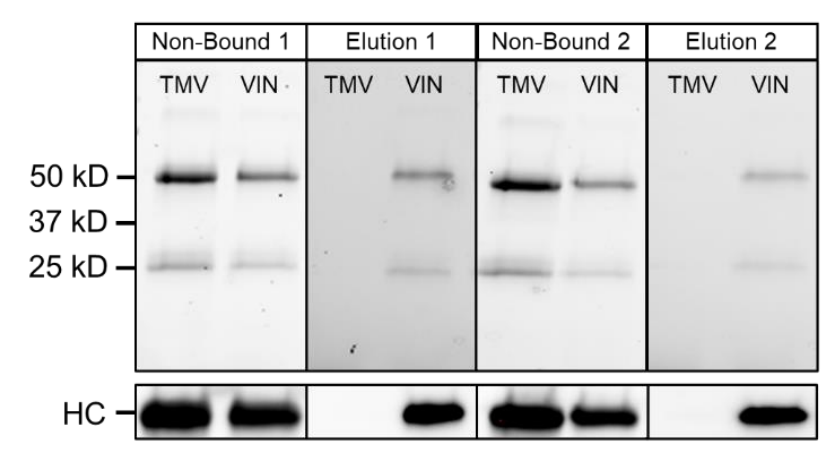

b

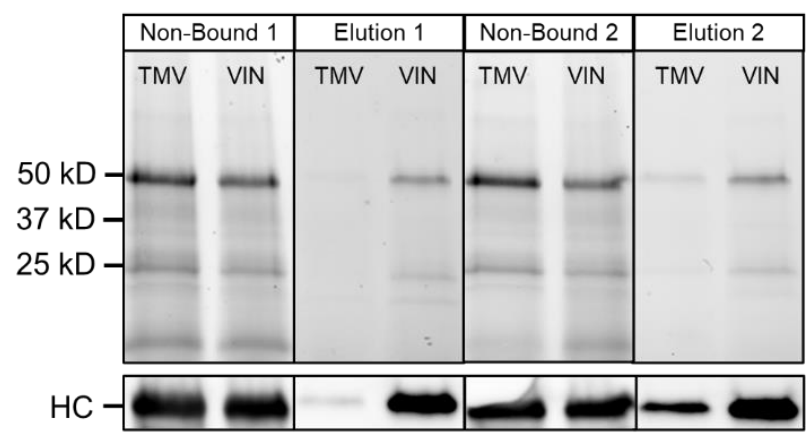

216 Figure 3. Use of silica sol-gel functionalized with entrapped plant virus-based immunosorbent

217 nanoparticles (VIN) to purify monoclonal antibodies. Reducing condition SDS-PAGE (upper) and

218 western blot (lower) results of the non-bound liquid sample after loading and low $\mathrm{pH}$ elution of

219 human immunoglobulin $\mathrm{G}$ for a first and second use cycle shown for a sample loading consisting 220 of human immunoglobulin G spiked into a solution of (a) clean PBS and (b) sterile-filtered 
221 Nicotiana benthamiana extract. Lanes are loaded with fixed volume. HC, heavy chain of the

222 human immunoglobulin G.

224 The small amount of hIgG in the elution for the wt-TMV negative control using $N$. benthamiana

225 extract (Figure 3b) indicates that the bind-and-elute functionality cannot be solely ascribed to

226 specific Protein A-Fc interactions. Additional negative controls of silica sol-gel synthesized with

227 bovine serum albumin and with no proteinaceous solution also corroborate these results (data not

228 shown). The hIgG recovered in the elution for the plant extract-containing sample condition

229 indicates some degree of non-specific binding interactions which may be present. Minor yellowish

230 coloration of the gel bead, which closely resembled the color of the loaded sample, after impurity

231 washing provides visual support for this observation (data not shown).

232 The second use cycle of hIgG recovery in elution indicates that the VIN functionality is preserved

233 over multiple uses. A three-week lag period between the gel synthesis and completion of the

234 second use cycle also provides additional support for the entrapment and stability of VIN in the

235 silica sol-gel environment. An increase in hIgG recovery for the second use cycle elution of the

236 wt-TMV bead and plant extract-containing sample condition is observed, which may indicate an

237 undesired behavior for performance such as incomplete hIgG elution from the first use cycle or an

238 additive effect of the non-specific binding mechanism between the first and second use cycle such

239 as what may be observed should a subset of the plant extract constituents accumulatively bind to

240 the silica sol-gel matrices.

241 The hIgG captured from the plant extract sample was recovered at $\sim 60 \%$ purity in both use cycles,

242 based on gel densitometry measurement. The SDS-PAGE results qualitatively support that the 
243 hIgG purity was significantly increased from the capture and elution procedure. A reduction in

244 recovery for the second use cycle was observed for hIgG in PBS ( $50 \%$ first elution) and in plant

245 extract ( $~ 80 \%$ first elution).

246 A substantial fraction of the hIgG sample loaded was not bound in either the VIN or wt-TMV

247 conditions. We attribute this to the excess loading concentration, which was used in this proof-of-

248 concept study to determine maximal elution given the employed gel synthesis and operational

249 configuration. Each gel bead was synthesized containing a total of $\sim 3 \mu \mathrm{g}$ VIN, which, based our

250 previous study of $\mathrm{VIN}$ in free solution recovering $1.5 \mathrm{mg} \mathrm{hIgG} / \mathrm{mg} \mathrm{VIN}$ in excess $\mathrm{hIgG}$

251 conditions, can be estimated to correspond to a binding capacity of $\sim 4.5 \mu \mathrm{g}$ hIgG. Each gel was

252 incubated in a liquid bath containing $20 \mu \mathrm{g}$ hIgG. Based on Bradford total soluble protein assay

253 results, we recovered $\sim 1.9 \mu \mathrm{g}$ hIgG from PBS for the first cycle elution from the immobilized VIN

254 sol-gel bead, corresponding to $\sim 10 \%$ hIgG recovery of the initial sample load. This binding

255 capability represents $\sim 42 \%$ of that of VIN in free solution.

\section{4. Discussion}

258 In this study, we report encouraging proof-of-concept results for novel VBN entrapment and

259 functionality within the pores of silica sol-gel matrices with broad applicability in protein

260 purification and biosensing. VIN-containing silica sol-gel beads were used to capture hIgG from

261 PBS or plant extract and elute the hIgG in a low pH environment for two consecutive use cycles

262 spanning approximately 30 days post-synthesis. The high surface area to volume ratio of the rod-

263 like VBNs used in this study proved to be an amenable geometry for pore entrapment with

264 considerable active binding site availability without the need for significant optimization. 
Future work to investigate the significance of VBN morphology on performance would be valuable for understanding the possible VBN silica gol-gel design space. Relevant characteristics for investigation include the geometry of the virion, either rod-like or icosahedral, the rigidity of the geometry, given that rod-like virions can be classified as stiff (e.g., tobacco mosaic virus) or flexible (e.g., potato virus X), and the size of the virion, which can be readily extended in rod-like virions through genome augmentation.

Non-specific binding interactions of the hIgG to the silica sol-gel were observed in this study, as observed for the wt-TMV and plant extract sample condition. We hypothesize that constituents of the plant extract bind to the silica sol-gel and in turn the extract-gel complex increases the nonspecific binding interactions with hIgG. Additionally, we hypothesize that the low $\mathrm{pH}$ elution conditions ( $\mathrm{pH} 2.5$ ) and the isoelectric points of the silica sol-gel matrix ( $\mathrm{pH} 2.0$ for silica) and hIgG (> pH 6.0) may generate a non-specific binding environment that could result in incomplete hIgG elution into the bulk liquid. This behavior was not observed in the experimental execution of this study but should be considered in the future. The isoelectric points of wt-TMV coat protein, $\mathrm{pH} \sim 4^{24}$, and VIN coat protein fusion, $\mathrm{pH} \sim 3.7$ as estimated using Expasy (web.expasy.org/compute_pi/), are both net negatively charged at the neutral gelation condition and not expected to non-specifically bind with the silica sol-gel matrix. We do suggest that future works more rigorously resolve concerns of non-specific binding interactions through optimization of silica sol-gel composition, perhaps considering doping (3-aminopropyl) triethoxysilane into the formulation, as has been successfully employed in previous studies ${ }^{25}$.

The reduction of effective binding capacity for silica sol-gel entrapped VIN was within the range of previously reported reductions in enzyme activity; silica sol-gel entrapped horseradish peroxidase and glucose-6-phosphate dehydrogenase enzymes were reported to exhibit specific 
288

289

290

291

292

293

294

295

296

297

298

299

300

301

302

303

304

305

306

307

308

309

310

activity of $73 \%$ and $36 \%$ of the specific activities of the free enzymes, respectively ${ }^{23}$. Further investigation is required to understand the relative contributions of protein activity modulation, such as from VIN exposure to gelation conditions or the internal pore environment $\mathrm{pH}$, and loss of accessibility of the active site, such as from diffusional limitations in the sol-gel matrix or partitioning of VIN into hIgG-inaccessible silica sol-gel matrix pores.

The findings of this study were consistent with the results of Kangasniemi and team for extended release of adenovirus from silica sol-gel in finding that the VBNs in silica sol-gel were stable for extended durations (weeks to months) and could be evenly distributed in the sol-gel postentrapment, as noted by the nearly linear VBN release profile during in vitro silica dissolution in their study ${ }^{16}$. The fundamental difference in observations was that Kangasniemi and team showed VBN activity to be retained for VBN that were released into free solution via silica dissolution, whereas this study reports VBN activity while entrapped within the silica sol-gel matrices. Our work also presents the first entrapment of plant virus-based VBN in silica sol-gel, which can be used in a wide variety of applications not amenable to mammalian virus-based VBN due to inherent advantages of safety (e.g., non-infectious to humans) and inexpensive and simple production.

The VIN silica sol-gel system presented in this study could serve as the foundation for a robust and reusable platform for biosensing. For example, the VIN system could be readily configured for immunosensing, in which the desired detection antibody would be first introduced to and fixed (via non-covalent bonding) in the VIN-entrapped gel matrix, allowing the subsequent addition of the desired analyte solution for sensing. The detection antibody could be removed via low $\mathrm{pH}$ elution and the same VIN-entrapped gel could be reused with a different detection antibody, as desired. This hypothesized example system may provide means for easy reuse and flexibility of 
311 sensing targets as well as an enhanced sensitivity over antibody-only systems due to the increased

312 sensing surface area of the structural scaffolding of the VIN. This hypothesis of the potential for

313 increased sensitivity is strongly supported by our recently reported results that VIN coupled to

314 magnetic particles could achieve $\sim 25 \mathrm{x}$ higher binding capacity of antibodies as compared to

315 current industry standards for affinity protein capture with magnetic particles on a per unit mass

316 basis $^{7}$.

317 Future works to further exploit the advantages of the highly tractable silica sol-gel chemistries for

318 more sophisticated geometries and fine-tuned pore architecture would be valuable. For example,

319 a previous study showed synthesis of bimodal pore distribution silica sol-gel in monolithic

320 nanoflow columns in which the smaller pores are used to entrap the ligand to functionalize the

321 column and the larger pores promote bulk flow and diffusion of the target molecule to the ligand

322 active site $^{26}$. This system architecture could be valuable for developing scale-down VBN-based

323 bioseparation or biosensing technologies.

\section{5. Acknowledgements}

326 This material is based upon work supported by NASA under grant or cooperative agreement award 327 number NNX17AJ31G, a NASA Space Technology Research Fellowship (NASA grant number 328 80NSSC18K1157), the Translational Research Institute through NASA Cooperative Agreement 329 NNX16AO69A, and the National Science Foundation (Grant number DMR - 1806366). Any 330 opinions, findings, and conclusions or recommendations expressed in this material are those of the 331 author(s) and do not necessarily reflect the views of the National Aeronautics and Space 
332 Administration (NASA), the Translational Research Institute for Space Health (TRISH), or the

333 National Science Foundation.

334

335 The illustrations were created in part using Biorender.com.

336

337 6. Disclosure Statement

338 The authors have no competing interests to report. 
340

341

342

343

344

345

346

347

348

349

350

351

352

353

354

355

356

357

358

359

360

\section{References}

1. Wen AM, Steinmetz NF. Design of virus-based nanomaterials for medicine, biotechnology, and energy. Chem Soc Rev. 2016;45(15):4074-4126.

doi:10.1039/C5CS00287G

2. Koudelka KJ, Pitek AS, Manchester M, Steinmetz NF. Virus-Based Nanoparticles as Versatile Nanomachines. Annu Rev Virol. 2015;2(1):379-401. doi:10.1146/annurevvirology-100114-055141

3. Ortega-Rivera OA, Pokorski JK, Steinmetz NF. A Single-Dose, Implant-Based, Trivalent Virus-like Particle Vaccine against "Cholesterol Checkpoint” Proteins. Adv Ther. March 2021:2100014. doi:10.1002/ADTP.202100014

4. Wang J, Yang M, Zhu Y, Wang L, Tomsia AP, Mao C. Phage nanofibers induce vascularized osteogenesis in 3D printed bone scaffolds. Adv Mater. 2014;26(29):49614966. doi:10.1002/adma.201400154

5. Tiu BDB, Kernan DL, Tiu SB, et al. Electrostatic layer-by-layer construction of fibrous TMV biofilms. Nanoscale. 2017;9(4):1580-1590. doi:10.1039/C6NR06266K

6. Uhde-Holzem K, McBurney M, Tiu BDB, et al. Production of Immunoabsorbent Nanoparticles by Displaying Single-Domain Protein A on Potato Virus X. Macromol Biosci. 2016;16(2):231-241. doi:10.1002/mabi.201500280

7. McNulty MJ, Schwartz A, Delzio J, et al. Affinity sedimentation and magnetic separation with plant-made immunosorbent nanoparticles for therapeutic protein purification. bioRxiv. November 2021:2021.11.05.467285. doi:10.1101/2021.11.05.467285 
361 8. Owens GJ, Singh RK, Foroutan F, et al. Sol-gel based materials for biomedical applications. Prog Mater Sci. 2016;77:1-79. doi:10.1016/J.PMATSCI.2015.12.001

9. Livage J, Coradin T. Encapsulation of Enzymes, Antibodies, and Bacteria. Handb Sol-Gel Sci Technol Process Charact Appl. July 2018:2909-2931. doi:10.1007/978-3-319-32101-

10. Nassif N, Bouvet O, Noelle Rager M, Roux C, Coradin T, Livage J. Living bacteria in silica gels. Nat Mater 2002 11. 2002;1(1):42-44. doi:10.1038/nmat709

11. Reátegui E, Kasinkas L, Kniesz K, Lefebvre MA, Aksan A. Silica-PEG gel immobilization of mammalian cells. J Mater Chem B. 2014;2(42):7440-7448. doi:10.1039/C4TB00812J

12. Ponamoreva ON, Kamanina OA, Alferov VA, et al. Yeast-based self-organized hybrid bio-silica sol-gels for the design of biosensors. Biosens Bioelectron. 2015;67:321-326. doi:10.1016/J.BIOS.2014.08.045

13. David AE, Yang AJ, Wang NS. Enzyme Stabilization and Immobilization by Sol-Gel Entrapment. Methods Mol Biol. 2011;679:49-66. doi:10.1007/978-1-60761-895-9_6

14. Kato K, Lee S, Nagata F. Efficient enzyme encapsulation inside sol-gel silica sheets prepared by poly-L-lysine as a catalyst. https://doi.org/101080/2187076420201747167. doi:10.1021/ACS.JPCB.6B10949 
382 16. Kangasniemi L, Koskinen M, Jokinen M, et al. Extended release of adenovirus from silica

383 implants in vitro and in vivo. Gene Ther 2009 161. 2008;16(1):103-110. doi:10.1038/gt.2008.142

385

386

387

17. Werner S, Marillonnet S, Hause G, Klimyuk V, Gleba Y. Immunoabsorbent nanoparticles based on a tobamovirus displaying protein A. Proc Natl Acad Sci U S A.

2006;103(47):17678-17683. doi:10.1073/pnas.0608869103

18. Kuo SY, Lin YC, Lai YC, et al. Production of fluorescent antibody-labeling proteins in plants using a viral vector and the application in the detection of Acidovorax citrulli and Bamboo mosaic virus. PLoS One. 2018;13(2). doi:10.1371/journal.pone.0192455

19. Giritch A, Marillonnet S, Engler C, et al. Rapid high-yield expression of full-size IgG antibodies in plants coinfected with noncompeting viral vectors. Proc Natl Acad Sci. 2006;103(40):14701-14706. doi:10.1073/PNAS.0606631103

20. Bruckman MA, Steinmetz NF. Chemical modification of the inner and outer surfaces of Tobacco Mosaic Virus (TMV). Methods Mol Biol. 2014;1108:173-185. doi:10.1007/978$1-62703-751-8 \_13$

21. Lam P, Gulati NM, Stewart PL, Keri RA, Steinmetz NF. Bioengineering of Tobacco Mosaic Virus to Create a Non-Infectious Positive Control for Ebola Diagnostic Assays. Sci Reports 2016 61. 2016;6(1):1-8. doi:10.1038/srep23803

22. Zeno WF, Hilt S, Aravagiri KK, et al. Analysis of lipid phase behavior and protein conformational changes in nanolipoprotein particles upon entrapment in sol-gel-derived silica. Langmuir. 2014;30(32):9780-9788. doi:10.1021/la5025058 
403 23. Bhatia RB, Brinker CJ, Gupta AK, Singh AK. Aqueous sol-gel process for protein

404 encapsulation. Chem Mater. 2000;12(8):2434-2441. doi:10.1021/cm000260f

405

24. OSTER G. THE ISOELECTRIC POINTS OF SOME STRAINS OF TOBACCO

406 MOSAIC VIRUS. J Biol Chem. 1951;190(1):55-59. doi:10.1016/S0021-9258(18)56044-0

407

25. Hodgson RJ, Chen Y, Zhang Z, et al. Protein-doped monolithic silica columns for capillary liquid chromatography prepared by the sol-gel method: Applications to frontal affinity chromatography. Anal Chem. 2004;76(10):2780-2790. doi:10.1021/ac0352124 approach for the development of sol-gel derived monolithic silica enzyme reactor 